\title{
Réponse physiologique au stade juvénile du génotype R405-2000 de cotonnier (Gossypium hirsutum L.) au déficit hydrique induit par le polyéthylène glycol
}

\author{
Tanoh H. Kouakou ${ }^{1 *}$, Mongomaké Kone ${ }^{1}$, Daouda Kone ${ }^{2}$, Yatty Justin Kouadio ${ }^{(1)} \&$ Michel Zouzou ${ }^{2}$ \\ ${ }^{1}$ Laboratoire de Biologie et Amélioration des Productions Végétales, UFR Sciences de la Nature, Université d'Abobo-Adjamé, \\ 02 BP 801 Abidjan 02, Côte d'lvoire. \\ ${ }^{2}$ Laboratoire de Physiologie Végétale, UFR Biosciences, Université de Cocody, 22 BP 582 Abidjan 22, Côte d'Ivoire. \\ 'Auteur pour les correspondances (E-mail: tanohilaire@yahoo.fr) \\ Reçu le 12-04-2007, accepté le 14-02-2008.
}

\begin{abstract}
Résumé
L'effet du stress hydrique osmotique induit par le polyéthylène glycol (PEG) a été étudié sur la germination et la croissance in vitro chez le cotonnier. Cinq concentrations différentes de PEG ont été testées et les résultats ont montré que le PEG agit négativement sur la germination des graines. La longueur des racines et des tiges diminue avec les concentrations élevées de PEG dans le milieu de culture. La croissance des feuilles est inhibée à partir de $15 \mathrm{~g} / \mathrm{l}$ de PEG dans le milieu de culture. Les parties aériennes ont été plus affectées par l'effet du PEG que les parties racinaires. Le flétrissement des plantules est observé à partir de $35 \mathrm{~g} / \mathrm{l}$ de PEG dans le milieu de culture.
\end{abstract}

Mots clés : cotonnier, culture in vitro, PEG, déficit hydrique

\begin{abstract}
Physiological response of cotton (Gossypium hirsutum L.) genotype R405-2000 at the youthful stage to water deficit induced by polyethylene glycol

The effect of water stress induced by polyethylene glycol (PEG) has been studied on germination and seedling growth of cotton. Five different concentrations of PEG have been tested and the results showed that PEG concentrations negatively influence seeds germination. The length of roots and stems decreases with high concentrations of $P E G$ in medium culture. The growth of leaves is inhibited from $15 \mathrm{~g} / \mathrm{L}$ of PEG in medium culture. Aerial parts have been more affected by water stress than the roots. Plantlet whithering is observed from $35 \mathrm{~g} / \mathrm{L}$ of PEG in medium culture.
\end{abstract}

Key words: cotton, culture in vitro, PEG, water deficit

\section{Introduction}

Le cotonnier est une plante cultivée dans les zones tropicales semi-arides pour ses fibres qui constituent la matière première principale en industrie textile. Gossypium hirsutum L. est l'espèce la plus cultivée et fournit près de $95 \%$ de la production mondiale de coton (ICA, 1992).
Les graines sont une source importante de protéines pour l'alimentation humaine et animale (Munro, 1987).

La Côte d'Ivoire, malgré la crise socio-politique, produit près de 400.000 tonnes de coton-graines, se plaçant ainsi au cinquième rang des producteurs africains au Sud du Sahara après le Burkina Faso, le Mali, le Tchad et le Bénin 
(Kouakou, 2003). Le coton représente une source de revenus très importante pour la Côte d'Ivoire où il occupe la troisième place des produits d'exportation après le cacao et le café. II procure à cet effet des revenus substantiels aux masses paysannes du Centre et du Nord du pays qui sont les régions cotonnières. Cependant, le développement de la culture du cotonnier est confronté aux problèmes de déficit hydrique et de maladies, ce qui provoque d'énormes pertes de production (Delhove et al., 1992). Toutefois, avec les produits phytosanitaires, la plupart des maladies du cotonnier sont traitées alors que le problème de déficit hydrique demeure. Le problème de sécheresse occupe donc et continuera d'occuper une très grande place dans la culture du cotonnier voire dans les chroniques agroéconomiques. Ainsi, les recherches concernant les mécanismes d'adaptation des plantes au déficit hydrique sont d'une importance primordiale.

Les mécanismes de résistance à la sécheresse impliquent des facteurs morphologiques, physiologiques et biochimiques à différents niveaux d'organisation de la plante. Selon May et Milthrope (1962) et Levitt (1972), les deux types de mécanismes d'adaptation à la sécheresse à envisager sont l'évitement et la tolérance. Ces mécanismes semblent conférer à la plante son aptitude à résister à un stress hydrique (Bouzoubaa \& El Mourid, 1999). D'après certains auteurs, les processus qui sont en relation avec la croissance et le développement des plantes sont influencés par le manque d'eau (Turner, 1986 ; Welbaum et al., 1990 ; Bradford, 1994 ; Duan et al., 2004; Sadeghian \& Yavari, 2004). A part le système racinaire et son rôle important dans l'absorption hydrique, les réponses morphologiques et physiologiques du système aérien, suite au déficit hydrique, constituent l'une des manifestations les plus évidentes de l'adaptation des plantes à la sécheresse (Monnevaux \& Nemmar, 1986). En outre, plusieurs travaux ont aussi montré que le polyéthylène glycol (PEG) est un inducteur de stress hydrique osmotique en culture in vitro (Biniek, 1994; Nepomuceno et al., 1998; Li et al., 2003; Robin et al., 2006; Rouhi et al., 2004; Turhan \& Baser, 2004).

Chez le cotonnier plusieurs génotypes sont résistants ou tolérants au déficit hydrique mais possèdent souvent une faible productivité (Leidi et al., 1993 ; Gadallah, 1995 ; Lacape et al., 1998 ;
Leidi et al., 1999). Le génotype R405-2000, mis au point et introduit récemment en Côte d'Ivoire, possède des caractères agronomiques intéressants avec une très bonne productivité. Depuis son introduction, aucune sécheresse sévère n'a sévi et donc son comportement vis-àvis du déficit hydrique n'a pas encore fait l'objet investigations bien menées.

C'est pourquoi, il nous a paru utile, d'évaluer la réponse physiologique du génotype R405-2000 de cotonnier au déficit hydrique. Ainsi, pour mettre en évidence les potentialités d'adaptation de ce génotype à la sécheresse, un stress hydrique a été induit en utilisant différentes concentrations de polyéthylène glycol (PEG) sur milieu gélosé. La réponse physiologique du génotype R4052000 de cotonnier vis-à-vis du déficit hydrique a été étudiée par la capacité de germination des graines et le développement de la plantule apprécié par la mesure de la croissance de la biomasse aérienne et racinaire en conditions contrôlées.

\section{Matériels et Méthodes}

Le matériel végétal est constitué de graines de cotonnier (Gossypium hirsutum L. cv. R 405-2000), originaire de Côte d'Ivoire. Ces graines proviennent de la région de Niéllé (Nord de la Côte d'Ivoire) et sont fournies par la CIDT (Compagnie Ivoirienne pour le Développement du Textile).

Les graines de cotonnier sont stérilisées dans l'éthanol $70 \%$ pendant une minute, puis dans l'hypochlorite de sodium (2,5\%) pendant 20 min et rincer trois fois à l'eau distillée stérile. Les graines stériles sont ensuite placées une à une dans des tubes à essais en verre de Pyrex de dimension $15 \times 2,2(\mathrm{~L} \times \varnothing$ extérieur en $\mathrm{cm})$ contenant $10 \mathrm{ml}$ de milieu de germination puis sont hermétiquement fermés avec des bouchons plastiques de dimension 3,8 × 2,5 ( $\mathrm{L} \times \varnothing$ extérieur en $\mathrm{cm}$ ). Le milieu de germination est constitué du milieu de base MS (Murashige \& Skoog, 1962) avec vitamines $\mathrm{B}_{5}$ (Gamborg et al., 1968) auquel on ajoute $30 \mathrm{~g} / \mathrm{l}$ de saccharose, $0,75 \mathrm{~g} / \mathrm{l}$ de $\mathrm{MgCl}_{2}$ et neuf solutions de concentrations croissantes $(0$, $10,15,20,25,30,35,40,50 \mathrm{~g} / \mathrm{l})$ de polyéthylène glycol (PEG 6000). La solidification des milieux se fait à ébullition par addition de 2,5 g/l de gelrite. Les milieux sont stérilisés à l'autoclave pendant 30 min sous 1 bar à $121^{\circ} \mathrm{C}$. Après inoculation, les tubes à essai sont placés dans une chambre de 
culture à une température de $28 \pm 2{ }^{\circ} \mathrm{C}$, sous une intensité lumineuse de 2000 lux et une photopériode journalière de 16 heures. Les tubes à essais contenant les graines sont ensuite placés à l'obscurité pendant deux jours pour initier la germination puis à la lumière sous une photopériode de $16 \mathrm{~h}$ pendant cinq jours. Des vitroplants cotonnier sont obtenus après sept jours de germination.

Un comptage a été réalisé pour dénombrer les graines qui ont germé et celles qui ont développé l'axe de la tige. Les longueurs de la radicule, de la tige et les dimensions (longueur et largeur) des feuilles ont été mesurées à l'aide d'une règle graduée. La biomasse totale et celle des parties aérienne et racinaire ont été déterminées par des pesées, après qu'elles aient été séchées dans une étuve à $60^{\circ} \mathrm{C}$ jusqu'à poids constant. Cette détermination du poids a été réalisée à l'aide d'une balance de précision.

L'analyse de variance suivie d'une comparaison de moyennes par le test de Newman-Keuls à un risque de $5 \%$ a été réalisée avec le logiciel Statistica 6.1 ont été réalisées pour déterminer l'effet du PEG sur la germination, la croissance et la biomasse. Dix graines de cotonnier ont été ensemencées par concentration de PEG avec trois répétitions.

\section{Résultats et discussion}

Le tableau I montre le résultat des quelques paramètres physiologiques du génotype R4052000 de cotonnier (Gossypium hirsutum L.) visà-vis du déficit hydrique induit par le PEG 6000 . Les résultats montrent que les différentes concentrations du PEG ont un effet significatif sur la germination in vitro des graines de cotonnier $(p<0,05)$. En effet, plus la concentration du milieu en PEG augmente plus le pouvoir germinatif diminue. Les taux de germination obtenus varient entre $92,57 \%$ pour le témoin et $15,33 \%$ pour le milieu le plus concentré en PEG. L'émergence de l'axe de la tige évolue de la même façon que le pouvoir germinatif mais avec des taux plus faibles. Ainsi, le pourcentage d'émergence de l'axe de la tige dans le milieu témoin est de $65 \%$, alors que le milieu le plus concentré en PEG a entraîné une inhibition presque totale de l'émergence de l'axe caulinaire (2\%). L'analyse statistique a permis de déceler un effet du PEG $(p<0,05)$ sur ce phénomène. Les différences observées pourraient s'expliquer par le fait que le PEG agit comme un agent osmotique en déshydratant les graines (Biniek, 1994; Almansouri et al., 2001). Ceci inhiberait l'hydrolyse des réserves nutritives de la graine donc la germination (Jan, 1986 ; Bewley \& Black, 1994; Dirik, 2000). II paraît clair que la germination et l'émergence des plantules ont été sévèrement affectées par le PEG. Djibril et al. (2005) ont obtenus des effets similaires avec le stress salin respectivement chez le palmier dattier. L'effet du stress hydrique par le PEG sur la croissance de la racine est très remarquable. En effet, une différence statistiquement significative $(p<0,05)$ a été notée entre les différentes concentrations de PEG. La longueur moyenne des feuilles est de $3,8 \mathrm{~cm}$ pour le témoin et de $1,3 \mathrm{~cm}$ pour la concentration la plus élevée de PEG, tandis que, la largeur moyenne des feuilles est de $2,7 \mathrm{~cm}$ pour le témoin et de $0,60 \mathrm{~cm}$ pour la concentration la plus élevée de PEG. Ce résultat montre la surface foliaire diminue avec les concentrations élevées de PEG. La hauteur moyenne de la tige à la fin de l'essai pour le témoin a été de $8,2 \mathrm{~cm}$ et de $1,5 \mathrm{~cm}$ pour la concentration la plus élevée de $\mathrm{PEG}$, alors que, pour la racine, cette longueur moyenne a été de $12,6 \mathrm{~cm}$ pour le témoin et de $4 \mathrm{~cm}$ pour la concentration de $50 \mathrm{~g} / \mathrm{l}$. Le rapport des longueurs racine sur tige a augmenté avec les concentrations élevées de PEG. Ce résultat montre que la partie aérienne est plus sensible à l'effet du PEG que la partie racinaire. La diminution de la croissance de l'appareil végétatif observée chez les plantules de cotonnier peut être expliquée par le fait que le PEG agit par augmentation de la pression osmotique du milieu, ce qui empêche l'absorption de l'eau par le système racinaire. Ceci entraîne par conséquent une réduction au niveau de la croissance de l'appareil végétatif comme l'ont montré Nepomuceno et al. (1998). Des effets similaires ont été remarqués sur la croissance de l'appareil végétatif des plantules d’arganier soumises à un stress hydrique en plein champ (Harrouni et al., 1995). Les mêmes résultats ont aussi été obtenus chez le persil et le cèdre du Liban respectivement par Biniek (1994) et Dirik (2000). Selon Bewley et Black (1994), le déficit en eau entraîne un retard dans la croissance végétale. Il se traduit par une réduction de la hauteur de la tige et une diminution de la surface foliaire (Aspinall, 1986). Ranjbarfordoei et al. (2000) a déduit que la croissance végétative et particulièrement l'expansion des feuilles sont 
sévèrement inhibées par le stress hydrique, les nouvelles feuilles se développent lentement et la sénescence des anciennes s'accélère. L'effet de la sécheresse s'exprime par un ralentissement progressif puis rapide de la croissance primaire puisque le déficit hydrique réduit la turgescence et par conséquent le pouvoir expansif des feuilles (Monnevaux \& Nemmar, 1986 ; Badiane et al., 2004). Par ailleurs, il est connu que la croissance est liée aux composantes de la production et du rendement. Au niveau de la production des biomasses aérienne et racinaire, l'analyse statistique a révélé un effet significatif de PEG $(p<0,05)$. Ainsi, les concentrations élevées de PEG entraînent une diminution du poids sec des parties aérienne et racinaire. D'après ces résultats, le rapport entre le poids sec racinaire et celui de la partie aérienne pour le témoin est de 0,164 alors que pour le milieu le plus concentré, il est de 0,054. II semble que la partie aérienne soit plus touchée par l'effet du PEG que la partie racinaire. En effet, chez l'oranger et le palmier dattier, les cultivars les plus résistants aux stress sont ceux qui limitent le plus efficacement possible l'accumulation des sels dans les feuilles (Greive \& Walker, 1983 ; Djibril et al., 2005). Du point de vue turgescence des organes, nous remarquons que la teneur en eau des plantules diminue avec les concentrations élevées de PEG. En effet, la teneur moyenne en eau par plantule est de $470 \mathrm{mg}$ pour la plantule témoin contre $50 \mathrm{mg}$ pour la plantule dont le milieu est le plus concentré en PEG. D'ailleurs, des symptômes de brunissement et de flétrissement des feuilles ont été observés sur les plantules à partir de la concentration $35 \mathrm{~g} / \mathrm{l}$. Ce même comportement a été observé chez la carotte (Biniek, 1994) et le blé (Almansouri et al., 2001), comportement qui était expliqué par une baisse du potentiel osmotique des feuilles. De même un dépérissement progressif puis rapide des plantules de cotonnier soumises au stress hydrique a été observé (Nepomuceno et al., 1998). Bouzoubaa et El Mourid (1999) rapportent que le poids frais, le poids sec et la composition minérale à l'exception de la proline subissent une diminution. L'accumulation de cet acide aminé est l'une des manifestations les plus remarquables du stress hydrique. Son rôle comme agent osmotique a été rapporté par plusieurs auteurs (Kramer, 1983 ; Bewley \& Black, 1994). La diminution de la croissance racinaire peut être expliquée par la subérisation des racines soumises au stress hydrique (Huang \& Reddman, 1995 ; Kefu et al., 2003). En revanche, il est reconnu que la résistance des végétaux à la sécheresse dépend du degré d'exploitation du sol par le système racinaire. Ainsi, des racines d'acacia à $30 \mathrm{~m}$ de profondeur que la superficie couverte par un seul spécimen d'arganier est de l'ordre de $67 \mathrm{~m}^{2}$ (Thierry, 1987). En ce qui concerne le cotonnier, son système racinaire couvre une surface qui peut atteindre $200 \mathrm{~m}^{2}$ (Parry, 1982). Donc, les plantes adultes réagissent vis-à-vis du stress hydrique par le développement du système radiculaire alors que dans notre cas, les plantules de cotonnier tendent à réduire la longueur des racines pour surmonter l'effet du stress hydrique provoqué par le PEG. Ceci permettrait une reprise des plantules dans les conditions idéales comme l'ont observé des auteurs chez de l'arganier (Harrouni et al., 1995 ; Zahri \& Harrouni, 1999). Nos résultats montrent que le déficit hydrique induit par le PEG influence la réponse physiologique du génotype R4052000 de cotonnier. R405-2000 semble donc être un génotype de cotonnier sensible au stress hydrique. Pour résister au déficit hydrique, les plantules de cotonnier réduisent considérablement la croissance de l'appareil végétatif. $\mathrm{Ceci}$, d'après plusieurs auteurs, n'est pas suffisant pour induire une tolérance au stress hydrique chez le cotonnier (Leidi et al., 1993 ; Nepomuceno et al., 1998 ; Sadras et al., 1996). 


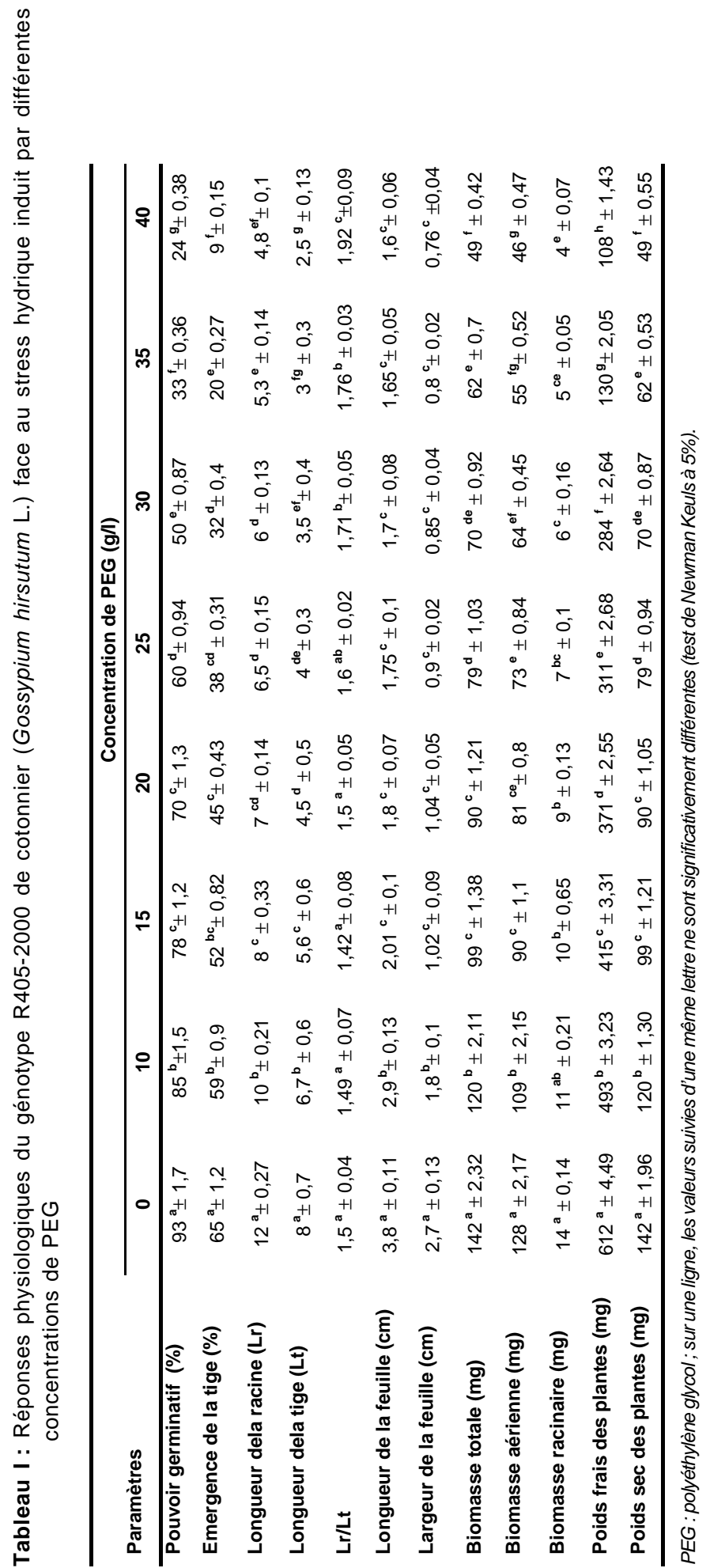

Sci. Nat. Vol. $5 N^{\circ} 1: 81-87$ (2008) 


\section{Conclusion}

L'étude du stress hydrique par le biais du PEG sur la germination in vitro du cotonnier révèle que le pouvoir germinatif des graines du cotonnier reste faible à la concentration de $50 \mathrm{~g} / \mathrm{l}$. Les plantules de cotonnier sont plus sensibles au déficit hydrique in vitro au niveau de la partie aérienne ; la concentration $35 \mathrm{~g} / \mathrm{l}$ entraîne un début de desséchement des feuilles. Les concentrations élevées de PEG entravent d'une façon significative la croissance de l'appareil végétatif. L'effet de PEG semble plus affecter la croissance végétative et la biomasse que la germination des graines. Le cultivar R405-2000 utilisé pour cette étude est donc un génotype sensible au déficit hydrique.

\section{Remerciements}

Les auteurs expriment leur gratitude à la Compagnie ivoirienne pour le développement du Textile (CIDT) pour la fourniture des graines de cotonnier.

\section{Références citées}

Almansouri M., Kinet J.M., \& Lutts S., 2001. Effect of salt and osmotic stresses on germination in durum wheat (Triticum durum Desf.). Plant and Soil 231: 243-254.

Aspinall D., 1986. Metabolic effects of water and salinity stress in relation to expansion of the leaf surface. Aust. J. Plant Physiol. 13: 59-73.

Badiane F., Diouf D., \& Siane D., 2004. Screening cowpea (Vigna unguiculata $L$.) varieties by inducing water deficit and RAPD analyses. Afr. J. Biotechn. 3(3): 174-178.

Bouzoubaa Z., \& El Mourid M., 1999. Étude comparative de quelques paramètres physiologiques de l'arganier sous plusieurs régimes hydriques. In: La Psoria UTET Periodici eds. Proceeding of first international conference on biodiversity and natural resource preservation. 13-14 May 1999. Ifrane (Maroc), pp. 31-34.

Bewley J.D., \& Black M., 1994. Cellular events during germination and seedling growth. In: N.Y. Plenum Press. Physiology of development and germination. New York, USA, pp. 147-197.

Biniek A., 1994. The influence of osmoconditioning in polyethylene glycol (peg 6000) on the germination and emergence of carrot and parsley seeds. Acta Hort. 371: 77-82. Bradford K.J., 1994.
Water stress and the water relations of seed development: A crit. Rev. Crop Sci. 34: 1-11.

Delhove G., Malamba N.L., \& Drion A., 1992. Maladies et ravageurs du cotonnier. In : Publication Agricole, AGCD Eds. Le cotonnier au Zaïre (volume 29), Bruxelles, Belgique. pp. 27-42.

Dirik H., 2000. Effet du stress hydrique osmotique sur la germination des graines chez les provenances de Cèdre du Liban (Cedrus libani A. Rich.) d'origine Turque. Ann. Forest Sci. 57: 371367.

Djibril S., Mohamed O.K., Diaga D., Diégane D., Abaye D.F., Sagna M. \& Borgel A., 2005. Growth and development of date palm (Phoenix dactylifera L.) seedlings under drought and salinity stresses. Afr. J. Biotech. 4 (9): 968-972.

Duan D., Liu X., Khan X., \& Gul B., 2004. Effects of salt and water stress on the germination of Chenopodium glaucum L., seed. Pak. J. Bot. 36 (4): 793-800.

Gadallah M.A., 1995. Effect of water stress, abscisic acid and praline on cotton plants. J. Arid Environ. 30 (3): 315-325.

Gamborg O.L., Miller R.A., \& Ojima K., 1968. Nutrient requirements of suspension cultures of soybean root cells. Exp. Cell Res. 50: 151-158.

Greive A.M., \& Walker R.R., 1983. Uptake and distribution of chloride sodium and potassium ions in salt-treated Citrus plants. Aust. J. Agric. Res. 34: 133-43.

Harrouni M., Zahr S., \& Elhemaid A., 1995. Transplantation des jeunes plantes d'arganier: effet combiné de techniques culturales et du stress hydrique. In: J. Libbey Eds. Actes du colloque international : la forêt face à la désertification " cas des arganeraies ». 26-28 octobre 1995. Agadir (Maroc). pp. 115-33.

Huang J., \& Reddman R.E., 1995. Salt tolerance of Hordeum and Brassica species during germination and early seedling growth. Can. J. Plant Sci. 75: 815-819.

ICA, 1992. International Cotton Advisory committee, cotton: world statistic, Washington D.C., USA, W.A. Geering Eds. 140 pp.

Jan K.P., 1986. Inhibition of Amaranthus caudatus seed germination by polyethylene glycol-6000 and abscissa acid and its reversal by ethephon or 1aminocyclopropane-1-carboxylic acid. Physiol. Plant. 67 (4): 588-597. 
Kefu Z., Hai F., San Z., \& Jie S., 2003. Study on the salt and drought tolerance of Suaeda salsa and Kalanchoe claigremontiana under isoosmotic salt and water stress. Plant Sci. 165: 837-844.

Kouakou T.H., 2003. Contribution à l'étude de l'embryogenèse somatique du cotonnier : évolution de quelques paramètres biochimiques au cours de la callogenèse et de la culture de suspension cellulaire, Thèse de Doctorat, Université de Cocody, Abidjan, Côte d'Ivoire, 144pp.

Kramer P.J., 1983. Water relation of plants. London, England: Academic Press Inc. 489 pp.

Lacape M.J., Wery J., \& Annerose D.J., 1998. Relationships between plant and soil water status in five field-grown cotton (Gossypium hirsutum L.) cultivars. Field Crops Res. 57 (1): 29-43.

Leidi E.O., Lopes M., \& Gutierrez J.C., 1993. Searching fort water stress in cotton genotypes: photosynthesis, stomatal conductance and transpiration. Photosynthetica 28 : 383-390.

Leidi E.O., Lopes M., Gorham J., \& Gutierrez J.C., 1999. Variation in carbon isotope discrimination and other traits related to drought tolerance in upland cotton cultivars under dryland conditions. Field Crops Res. 61: 109-123.

Levitt J., 1972. Responses of plants to environmental stress. New York, USA: Academic Press, 697pp.

Li M., Wang G., \& Lin J,. 2003. Application of external calcium in improving the PEG-induced water stress tolerance in liquorice cells. Bot. Bull. Acad. Sinica 44 (4): 275-284.

May L.H., \& Milthrope F.L., 1962. Drought resistance of corps plants. Fields Corp. Abstr. 15: 171-179.

Monnevaux P. \& Nemmar M., 1986. Contribution de l'étude de la résistance à la sécheresse chez le blé tendre (Triticum aestivum L.) et le blé dur (Triticum durum Desf.). Etude de l'accumulation de la proline au cours du cycle de développement. Agronomie 6: 583-590.

Munro J.M., 1987. Cotton. Trop. Agri. 2: 170-172.

Murashige T., \& Skoog F., 1962. A revised medium for rapid growth and bioassay with Tobacco tissue cultures. Physiol. Plant. 15: 473-497.

Nepomuceno A., Oostrerhuis D., \& Stewart J., 1998. Physiological Reponses of cotton leaves and roots to water deficit induced by polyethylene glycol. Env. Exp. Bot. 40: 29-41.
Parry G., 1982. Le cotonnier et ses produits (volume 30). Paris, France. Eds. G-P Maisonneuve \& Larose. $502 \mathrm{pp}$.

Ranjbarfordoei A., Samson R., Damme P., \& Lemeur R., 2000. Effects of drought stress induced by polyethylene glycol on pigment content and photosynthetic gas exchange of pistacia. Photosynthetica 38: 443-447.

RobinC., Shamsun-NoorL., \& GuckertA., 2006. Effect of potassium on the tolerance to PEG-induced water stress of two white clover varieties (Trifolium repens L.). Plant and Soil 120 (2): 153-158.

Rouhi V., Samson R., Damme P. van, \& Lemeur R., 2004. Effects of drought stress induced by PEG 6000 on leaf water status of one domestic (Amygdalus dulcis) and two wild almond ( $A$. lycioides and A. scoparia) species. Agric. Applied Biol. Sci. 69 (2): 239-241.

Sadras, V.O, Wilson, L.J., \& Lally D.A., 1998. Water deficit enhanced cotton resistance to spider mite herbovory. Ann. Bot. 82 (2): 273-286.

Sadeghian, S.Y., \& Yavari N., 2004. Effect of waterdeficit stress on germination and early seedling growth in sugar beet. J. Agron. Crop Sci. 190 (2): 138-144.

Thierry L., 1987. L'arganier au Maroc : sa description, ses méthodes de multiplication et son application en reforestation. Thèse d'ingénieur technique. Institut provençal d'enseignement supérieur agronomique et technique, Agadir, Maroc. 183 pp.

Turhan H., \& Baser I., 2004. In vitro and in vivo water stress in sunflower (Helianthus annuus L.). Helia 27 (40): 227-236.

Turner NC., 1986. Adaptation to water deficits: a changing perspective. Aust J. Plant Physiol. 13: 175-90.

Welbaum G.E., Tissaoui T., \& Bradford K.J., 1990. Water relations of seed development and germination in muskmelon (Cucumis melo L.). III- Sensitivity of germination to water potential and abscisic acid during development. Plant Physiol. 92: 1029-1037.

Zahri S., \& Harrouni M.C., 1999. Optimisation de la reprise après transplantation chez l'arganier (Argania spinosa L. Skeels). In: La Psoria UTET Periodici Eds. Proceeding of first international conference on biodiversity and natural resource preservation. 1314 May 1999. Ifrane (Maroc), pp. 63-68. 